\title{
Implementation of goal-directed therapy needs a boost, and it is called assisted fluid management
}

\author{
Paul A. van Beest ${ }^{1,2}$
}

Received: 11 May 2018 / Accepted: 14 May 2018 / Published online: 16 May 2018

๑) Springer Science+Business Media B.V., part of Springer Nature 2018

It is a fact of life: implementation of something new, anything new at all, on the work floor is challenging. Even when your new protocol is as evidence based as a new protocol can be, implementation demands perseverance. One of the reasons for this difficulty is that changing behavior without the presence of logistical, procedural, or technical errors on the work floor does not motivate clinical collectives to action [1]. To make behavioral and professional change possible clinicians should be taught about the why, what (how) and when a certain new protocol or device will be implemented. Repetitive teaching and communication is key.

An intriguing example of reproducible clinical finding that is still not embraced by everybody is central venous pressure (CVP) as a measure for the filling status or fluid responsiveness of a patient. Despite an impressive amount of literature that has been published over the last two decades on this matter, showing that CVP is not an adequate predictor for volume status or fluid responsiveness there are still colleagues out there who believe the exact opposite. Hence, the use of so called dynamic variables for volume status, i.e. stroke volume variation (SVV) or pulse pressure variation (PVV) is of yet not the golden standard.

Honest research may be hard work, implementing reproducible results can be even harder. Implementation of GoalDirected Therapy (GDT), which in part uses the abovementioned dynamic variables, at the operating room (OR) is no exception. Multiple studies [2-5] showed reduction in postoperative complications after use of GDT compared to controls. Also, the European Society of Anaesthesiology (ESA) incorporated GDT in Guidelines on non-cardiac

Paul A. van Beest

paul.van.beest@znb.nl

1 Department of Anesthesiology, Medical Center Leeuwarden, Henri Dunantweg 2, 8934 AD Leeuwarden, The Netherlands

2 Department of Anesthesiology, University Medical Center Groningen, University of Groningen, Groningen, The Netherlands surgery: cardiovascular assessment and therapy [6]. Today, based on the accumulating evidence underlining the advantages of goal-directed fluid therapy, one could think that broad implementation of GDT in the OR setting is a piece of cake. Unfortunately, nothing could be further from the truth! Disbelieve, lack of education and workload may stand in the way of adherence to and implementation of GDT protocols [7].

Behold! In this journal's issue, a new clinical decision support system is introduced [8]. This system, Assisted Fluid Management (AFM) was designed to help ease some of the workload associated with GDT protocol implementation. As described by Joosten et al. [8] the system can suggest fluid bolus administration and continuously re-assess the patient's needs for further intra operative fluid administration. In short, the researchers compared a cohort of historical patients in whom a SVV based GDT protocol was used (control group) with prospectively included patient in whom GDT was assisted by AFM (AFM group). The researchers hypothesized that the use of AFM would result in a higher percentage of time spent under SVV cut-off value of $13 \%$. In other words, the patient would be less fluid responsive which probably would be accompanied by a better cardiac performance. In addition, as a result of better GDT protocol adherence the number of complications could be reduced [8].

The null hypothesis was not rejected: during surgery patients in the AFM group spent significantly more time under the SVV cut-off value of $13 \%$ while these patients received approximately $400 \mathrm{ml}$ less fluids. In addition, the mean arterial pressure (MAP) in the AFM group was higher. In contrast, significantly more patients in the control group received noradrenaline. Finally, despite the very high $(97 \%)$ adherence to the AFM system in the clinical setting no differences in complications between groups was observed.

What does this study tell us about AFM and its influence on decision making in hemodynamic monitoring during 
surgery? Well, a definitive answer whether we should implement this system in our daily practice is not provided.

First, what was really compared here? Maybe we are looking at the comparison of two different GDT protocols, i.e. a SVV based protocol (control group) versus a SV target based protocol (AFM group). Indeed the system seems to rely on SVV to guide boli. However, the AFM system had a user-configurable target (standard 15\%) for SV response after fluid bolus. One could argue that the latter is a more physiologically driven approach resulting in better cardiac performance. Of note, underlying algorithms must be explicable and robust so the clinician can be convinced of its use. The fact that the patients in the AFM group received less fluids is mainly the result of a lower maintenance crystalloid volume. Interestingly, this could be an argument to set a new standard for maintenance volume: zero.

Second, the compliance to the GDT protocol in the historical group (control group) is not known. Hence, a true comparison is not possible. Also, it cannot be excluded that the involved clinicians partially fell prey to investigators bias: the AFM system relieves the clinician of some workload which can be very tempting at times. The high compliance presented may not be entirely reproducible. However, the result of the different methods is the significant difference in time under the SVV cut-off value and that counts! A better performance in GDT protocol adherence is an important step to a more patient tailored approach with an overall reduction of perioperative reduction of perioperative complications in high surgical patients.

As mentioned by the investigators themselves, outcomes should be interpreted with caution given the difference in baseline POSSUM physiology score. Also, sample size is relatively small.

Even in an environment where GDT has been widely adopted AFM made a positive difference. The system proved to be reliable in a clinical setting: the compliance to the system was high and non-effective recommended boli were practically non-existent. Albeit the shortcomings of the study, its results are very encouraging. This study deserves continuation, preferably in the form of a randomized controlled trial.

In conclusion, efforts to improve perioperative hemodynamic monitoring with better outcomes as the ultimate goal can be supported by AFM. Nevertheless, teaching efforts on the use of GDT itself will probably be necessary for a long time to come.

\section{Compliance with ethical standards}

Conflict of interest The author has given several presentations on GDT protocols and it's implementation supported by Edwards Lifesciences devices.

\section{References}

1. Cook DJ, Montori VM, McMullin JP, Finfer SR, Rocker GM. Improving patients' safety locally: changing clinician behaviour. Lancet 2004;363:1224-30.

2. Gan TJ, Soppitt A, Maroof M, El-Moalem H, Robertson KM, Moretti E, Dwane P. Goal-directed intraoperative fluid administration reduces length of hospital stay after major surgery. Anesthesiology 2002;97:820-6.

3. Scheeren TWL, Wiesenack CH. Goal-directed intraoperative fluid therapy guided by stroke volume and its variation in high-risk surgical patients: a prospective randomized multicenter study. J Clin Monit Comput. 2013;27:225-33.

4. Benes J, Giglio M, Brienza N, Michard F. The effects of goaldirected fluid therapy based on dynamic parameters on post-surgical outcome: a meta-analysis of randomized controlled trials. Crit Care. 2014;18(5):584.

5. Michard F, Giglio MT, Brienza N. Perioperative goal-directed therapy with uncalibrated pulse contour methods: impact on fluid management and postoperative outcome. Br J Anaesth. 2017;119(1):22-30.

6. Task Force Members, et al. 2014 ESC/ESA guidelines on noncardiac surgery: cardiovascular assessment and management: The Joint Task Force on non-cardiac surgery: cardiovascular assessment and management of the European Society of Cardiology (ESC) and the European Society of Anaesthesiology (ESA). Eur J Anaesthesiol. 2014;31(10):517-73.

7. Cannesson M, Ramsingh D, Rinehart J, Demirjian A, Vu T, Vakharia S, Imagawa D, Yu Z, Greenfield S, Kain Z. Perioperative goal-directed therapy and postoperative outcomes in patients undergoing high-risk abdominal surgery: a historical-prospective, comparative effectiveness study. Crit Care. 2015;19:261.

8. Joosten A, Hafiane R, Pustetto M, Van Obbergh L, Quackels TH, Buggenhout A, Vincent JL, Ickx B, Rinehart J. Practical impact of a decision support for goal-directed fluid therapy on protocol adherence: a clinical implementation study in patients undergoing major abdominal surgery. J Clin Monit Comput. 2018. 